Revue

Revue de l'histoire des religions

de Ihistoire des religions

\title{
Quirinus, un ancien dieu tonnant? Nouvelles hypothèses sur son étymologie et sa nature primitive
}

Was Quirinus a thunder-god? New hypothesis about his etymology and his primitive nature

\section{Patrice Lajoye}

\section{(2) OpenEdition}

\section{Journals}

Édition électronique

URL : https://journals.openedition.org/rhr/7573

DOI : $10.4000 /$ rhr.7573

ISSN : 2105-2573

\section{Éditeur}

Armand Colin

\section{Édition imprimée}

Date de publication : 1 juin 2010

Pagination : 175-194

ISBN : 978-2200-92656-4

ISSN : 0035-1423

\section{Référence électronique}

Patrice Lajoye, «Quirinus, un ancien dieu tonnant ? Nouvelles hypothèses sur son étymologie et sa nature primitive », Revue de l'histoire des religions [En ligne], 2 | 2010, mis en ligne le 01 juin 2013, consulté le 21 septembre 2021. URL : http://journals.openedition.org/rhr/7573 ; DOI : https://doi.org/ 10.4000/rhr.7573 


\section{Quirinus, un ancien dieu tonnant? Nouvelles hypothèses sur son étymologie et sa nature primitive}

La découverte il y a maintenant plus de vingt ans d'une série d'inscriptions consacrées à Hercule Quirinus permet de remettre en cause les hypothèses de Georges Dumézil sur ce dieu prétendu agraire qui aurait représenté à Rome la troisième fonction indo-européenne. Un examen linguistique de son nom archaïque, Queirinus, peut permettre d'en faire un ancien dieu tonnant, dont la mythologie de Romulus conserve quelques traces.

\section{Was Quirinus a thunder-god?}

New hypothesis about his etymology

and his primitive nature

More than twenty years ago, the discovery of a series of inscriptions dedicated to Hercules Quirinus allows to call into question the hypotheses of George Dumézil on this agricultural alleged God who would have represented the third indo-european function at Rome. A linguistic exam of its archaic name, Queirinus, can allow to make an ancient thundering God, Romulus's mythology of which keeps some traces. 
On peut dire que le problème de Quirinus a été une des questions qui ont le plus retenu l'attention de Georges Dumézil : il lui a consacré trois livres, ainsi que de nombreuses pages disséminées ailleurs dans son œuvre ${ }^{1}$. L'hypothèse de Georges Dumézil est simple : il a existé à Rome une triade pré-capitoline, composée de Jupiter, Mars et Quirinus, ce dernier dieu étant déjà fort peu connu des Romains de l'époque impériale. Jupiter étant le grand dieu céleste représentatif de la première fonction indo-européenne, Mars un dieu guerrier appartenant donc à la deuxième fonction ${ }^{2}$, reste Quirinus qui, pour entrer dans le cadre de l'idéologie tripartite doit obligatoirement appartenir à la troisième fonction. L'auteur fait alors appel à une série d'arguments montrant les aspects agraires et pacifiques de Quirinus.

\section{QUIRINUS AGRAIRE}

Selon une hypothèse déjà ancienne, Quirinus serait issu de *couirino- (*co-uirio- + suffixe) et serait donc le dieu de l'ensemble des hommes ${ }^{3}$. Or, note Georges Dumézil, ce serait un fait chez les Indo-Européens que les dieux de la troisième fonction aiment à vivre parmi les hommes, comme l'aurait fait Quirinus sous le nom de Romulus ${ }^{4}$. De même, le fait que Romulus-Quirinus soit un jumeau serait à mettre en rapport avec les jumeaux indiens de troisième fonction, les Aśvin 5 .

En conséquence, Quirinus ne peut être qu'un dieu pacifique, ce qui expliquerait quelques vers d'Ovide sur les Robigalia, fête au cours de laquelle le flamine de Quirinus officie, et où on demande

1. Dumézil, 1941; 1944; 1948 (le volume III de cette série, Naissance d'archanges, 1945, Paris, Gallimard, étant un peu marginal). La synthèse de ces données est faite dans Dumézil, 1949 et surtout 1966. Dumézil reviendra souvent sur la question : Dumézil, 1995, p. 296-297, 2003, p. 447-456 et 1996, p. 160-166. Il a été suivi très tôt par Basanoff, 1942, p. 14-18; et l'est encore par Schilling, 1999 ou même Sergent, 1995, p. 331-332.

2. Dumézil nie tout aspect agraire à Mars. Nous reviendrons sur ce problème.

3. Basanoff, 1942, p. 17.

4. Dumézil, 1966, p. 251.

5. Ibid., p. 252. 
à Robigus d'aller rouiller les armes plutôt que les récoltes ${ }^{6}$. De fait, le flamine de Quirinus semble n'officier que lors de fêtes bien pacifiques dont certaines sont agraires: Robigalia, donc, Consualia, Larentalia. Fêtes que l'on peut compléter par les Quirinalia qui clôturent les Fornacalia. On en vient alors à l'opposition quirites/ milites, qu'on peut joindre au dossier en supposant que les Quirites ont un rapport étymologique avec Quirinus. Les Quirites seraient, et de façon très ancienne selon Georges Dumézil, des civils?.

Vient alors le fameux témoignage de Servius, commentant l'Énéide, faisant de Quirinus un «Mars tranquille», par opposition à Gradivus, qui serait un "Mars déchaîné ». Quirinus est le Mars qui préside à la paix, ce que confirme bien l'existence d'un collège de Salii de Quirinus, créé pour mettre fin à un conflit ${ }^{8}$. Ces arguments poussent Georges Dumézil à surmonter l'obstacle de l'interprétation grecque de Quirinus par Enyalios, épithète d'Arès, en faisant appel à une simplification excessive due à l'assimilation à Romulus, dont le caractère guerrier ne fait pas de doute. Le phénomène inverse se serait produit à Rome, où Quirinus pacifique se rapproche de Janus9. Georges Dumézil remarque aussi que le flamine de Quirinus, selon Aulu Gelle (7, 7, 7), intervient dans les Larentalia, fête bien évidemment de troisième fonction. Cette cérémonie aurait été instituée par une courtisane, Acca Larentia, qui avait accepté de passer une nuit dans le temple d'Hercule et en serait ressortie riche. Mais elle serait aussi la femme du berger qui avait recueilli Romulus et Remus.

\section{QUIRINUS GUERRIER}

Allégés des nombreuses digressions comparatistes sur les Aśvin ou sur Nerthus, les arguments de Georges Dumézil en faveur d'un Quirinus appartenant à la troisième fonction sont finalement assez peu nombreux : rapprochement étymologique avec les Quirites, participation du flamine de Quirinus à des fêtes agraires, Quirinus dieu de paix.

\footnotetext{
6. Ovide, Fastes, 4, 911-932.

7. Dumézil, 1966, p. 259.

8. Ibid., p. 275.

9. Ibid., p. 264-265.
} 
En 1981, Danielle Porte a tenté de revoir la question dans un long article détaillé. Pour elle, Quirinus reste un dieu guerrier, qui possède ses Salii, mais aussi des armes, des boucliers sacrés, des prêtres fétiaux, chargés de déclarer la guerre ${ }^{10}$. De même, il lui semble, à raison, que les Grecs n'ont pas donné à la légère le nom d'Enyalios à Quirinus. Que l'interpretatio ait ses limites comme le signale Georges Dumézil, c'est vrai, mais pas à ce point ${ }^{11}$. De fait, il semble que les Grecs aient eu du mal à dissocier Mars de Quirinus, au point que Denys d'Halicarnasse se demande s'il ne s'agirait pas d'un même dieu ${ }^{12}$. En définitive, Danielle Porte démonte point par point l'argumentation agraire de Georges Dumézil, jusque dans l'organisation des fêtes auxquelles Quirinus semble être lié (Larentalia, Robigalia, Consualia et bien sûr Quirinalia). Si pour elle, Quirinus est plus largement un dieu des citoyens dans leur ensemble, il nous semble préférable d'y voir un probable ancien dieu guerrier.

On se retrouverait donc avec une sorte de doublon de Mars, lequel a longtemps été vu lui aussi comme une divinité primitivement agraire.

\section{À LA RECHERCHE DE DOCUMENTS NOUVEAUX}

En fait, pour trancher entre les deux hypothèses, il faut partir à la recherche de documents nouveaux ou méconnus, documents issus de l'épigraphie et de la numismatique.

Commençons par l'épigraphie. On peut se détourner d'emblée des quelques rares inscriptions consacrées à Quirinus seul : elles n'apportent rien au dossier. Reste une dédicace offerte par un affranchi à Rome :

$\mathrm{P}$ (ublius) Cornelius $\mathrm{P}($ ubli) 1(ibertus)/Gaipor $\mathrm{P}$ (hiladelphus)/ I(ovi) Q(uirino) et $\mathrm{Ma}$ (rti) s(acrum) ${ }^{13}$.

10. Porte, 1981, p. 305. C'est volontairement que je ne ferai appel qu'à cet article, parmi tous les opposants à la thèse de Georges Dumézil, car il a le mérite de faire un tour complet de la question en n'utilisant que des éléments d'information romains. L'unique défaut que l'on peut relever dans l'argumentation de Danielle Porte est qu'elle tende à voir en Quirinus une simple épithète, ce qui n'est pas le cas. En 1984, il semble que Georges Dumézil préparait une réponse à cet article : Dumézil, 1996, p. 165. Il n'a pas eu le temps de la publier.

11. Porte, 1981, p. 311.

12. Ibid.

13. CIL VI, 30914 (p. 3758) = CIL I, 996 (p. 965) = AE 2000, 131. 
Une hypothèse précédente faisait mention de « I(ano ?) Q(uirino?) et $\mathrm{Ma}(\mathrm{rti}$ ?) s(acrum?) ».

On remarquera l'ordre étonnant de la triade : Quirinus vient avant Mars! C'est peut-être ce qui permet d'interpréter la seconde inscription, provenant de Castelvecchio Subequo, dans le Samnium :

a : [Io] vi Cyrii [// C(ai) Tati Max(imi)

$\mathrm{b}:[\mathrm{Io}]$ vi Quirin [0 $\mathbf{0}^{14}$

Cette dédicace avait été écartée assez sèchement par Georges Dumézil ${ }^{15}$. Mais en tenant compte de l'inscription de Rome, on peut suggérer :

a : [Io] vi Cyri [no et Marti...// C(ai) Tati Max(imi)

b : [Io] vi Quirin [o et Marti...

La graphie Cyrino s'explique grâce à un dossier qui a vu le jour au début des années 1980, et dont la publication s'est étalée dans le temps. Georges Dumézil n'a pu prendre connaissance de ces inscriptions, et cela est bien dommage car elles révolutionnent la question. À Sulmona, toujours dans l'ancien Samnium, on a découvert un sanctuaire d'Hercule bien particulier : celui-ci contenait encore une série de dédicaces et de graffiti votifs :

* [--- f?]ac ei/[--- Herc] ules Quirine s[ancte? ---]/[---] sere saep[e ? --- $] /[---]$ ucem $[\& 16$

* Augustis et sancte Curin [e]/digna paramus spectat [a]/nam debita solvere vota [decet]/numenque sacratum/ecce venit felixque pat [et]/pristina vota feren [tes]/[veni] mus scimus qui fuerint ipse $[\mathrm{met}] /$ isdem sei (!) voves $\mathrm{H}$ (erculi) $\mathbf{C}$ (urino) $\mathrm{V}($ ictori ?)/si mi somnia exseri[t? $]^{17}$

* L (ucius) Albius L(uci) 1(ibertus)/Eros/scalpto(r) statuarius/ Her(culi) Cur(ino) v(otum) s(olvit) 1(ibens) m(erito) ${ }^{18}$

*C(aius) Nonius L(uci) f(ilius) Serg[ia ---]/e munici[p]io Sulmone $\mathrm{p}[$ romisit]/miles Herculi Curino sei sal[vus e]/castris rediset vot[a ---]/verem et vitulu[m]/et votis dam[natus]/[a] dest ${ }^{19}$

14. CIL IX, $3303 a$ et $b$.

15. Dumézil, 1966, p. 265, n. 1: «Quant à un Jupiter Quirinus (CIL. IX 3303), il est plus qu'improbable ».

16. AE 1981, 285.

17. $\mathrm{AE} 1981,282=\mathrm{AE} 1983,328$

18. AE $1980,376=$ AE 1984, 325

19. AE 1981, 283 
* Cn(aeus) Domitius/Cn(aei) 1(ibertus) Amemptus/Herc(uli) Cur(ino) v(otum) $s$ (olvit) ${ }^{20}$

* Ades/hic fuisti/[-- Hercul ?]ei Corino ${ }^{21}$

* [--- S] aturnie Queir[ine ---]// [---] S in Ka[lendis ${ }^{22}$

* [---] sei Hercules Queirinu[s ---]/[---] s est reliquis praes [--- $]^{23}$

Cette série daterait vraisemblablement de la fin du II siècle avant J.-C. Elle est donc très ancienne, plus ancienne que tous les textes étudiés jusqu'ici. Ce qui est neuf est d'abord l'assimilation à Hercule, mais aussi la grande diversité d'orthographes, laquelle peut avoir plusieurs causes ${ }^{24}$. On possède donc d'abord un Hercule Quirinus, mais aussi un Hercule Curinus, voire Corinos (graphie peut-être fautive). Cette variante peut venir soit d'une transcription grecque (Quirinus s'écrit Quirinus par Curia. Reste Queirinus, graphie qui n'est pas fautive puisqu'elle est répétée deux fois. Elle a une grande importance pour l'étymologie du mot.

\section{Étymologie}

Les anciens étaient déjà très partagés sur l'étymologie de Quirinus : les uns en faisaient un dieu originaire de la ville sabine de Cures, les autres tiraient son nom du vocable sabin de la lance. Chez les modernes, on peut noter la tentative de Cook, qui, en 1904, avait noté une analogie entre Quirinus et quercus ( « chêne ») et suggéré par conséquent que Quirinus puisse être étymologiquement un «dieu-chêne ». Cependant, il pensait que Quirinus était construit de la même manière que le grec prinos : "yeuse, chêne vert, houx », et que l'assonance avec quercus n'était donc qu'accidentelle $\mathrm{e}^{25}$. L'hypothèse est reprise par Frazer, qui la simplifie en admettant un rapport explicite, quoique nuancé, entre Quirinus et

20. AE 1989,238

21. AE 1981, 290

22. AE 1981, 286

23. AE 1981, 284

24. L'assimilation à Hercule doit peut-être être rapprochée de la participation du flamine de Quirinus aux Larentalia, fête instituée, on l'a vu, par le testament d'une courtisane qui avait passé une nuit dans le temple d'Hercule. Y a-t-il eu par le passé une tentative avortée d'assimilation à Hercule de Quirinus?

25. Cook, 1904 , p. 368 , n. 22. Le sabin curis serait alors, selon lui, pour « lance en chêne ». 
quercus $^{26}$. Enfin, Reiche avait proposé de faire dériver le nom de *quernus, « chêne », sans pour autant pouvoir démontrer son hypothèse ${ }^{27}$.

Plus récemment, Radke a proposé une étymologie basée sur un hypothétique *quirium : "laboureur », hypothèse trouvant un renfort dans le fait que Romulus a délimité le site de la future Rome avec une charrue ${ }^{28}$. Cependant son hypothèse n'a pas été suivie, et de fait, ni Quirinus, ni les Quirites ne sont des laboureurs.

À la suite de Kretschmer, beaucoup admettent donc un dérivé de *co-uiros, «l'assemblée des hommes », donnant *co-viria pour curia (étymologie étayée par le volsque couehriu), *co-uiri-tes pour les Quirites et *co-uiri-nos pour Quirinus ${ }^{29}$. Cette étymologie a été acceptée par la plupart des auteurs postérieurs ${ }^{30}$, à l'exception par exemple de A. Ernout et A. Meillet, qui ne l'admettent que pour curia $^{31}$. Cependant, cette «assemblée des hommes» doit se comprendre comme étant l'assemblée de tous les hommes, et non seulement des civils ${ }^{32}$. Georges Dumézil lui-même l'admet : quirites et milites sont les mêmes hommes ${ }^{33}$. D'une manière générale, *viro- désigne, dans toutes les langues indo-européennes où il s'est conservé, l'homme mâle, viril ${ }^{34}$.

Cependant, la forme Queirinus attestée à Sulmona s'avère gênante pour cette hypothèse. Il faut vraisemblablement chercher l'étymologie d'un terme du type *Querinos, puis *Queirinos ${ }^{35}$, qui aurait alors subi l'attraction de curia et quirites. Ensuite, le passage de Queirinus à Quirinus se fait naturellement, le latin réduisant normalement, dès la première moitié du II ${ }^{\mathrm{e}}$ siècle avant J.-C., la diph-

26. Frazer, 1935, p. 157, et p. 349 , n. 584.

27. Klio, XXI, 1926, p. 74-78.

28. Radke, 1981.

29. Kretschmer, 1920

30. Voir par exemple, Haudry, 2006, p. 48, qui note cependant des difficultés phonétiques qualifiées de surmontables.

31. Ernout et Meillet, 1932, sv. curia et quiris.

32. Prosdocimi, 1996. C'est ce que note aussi Dominique Briquel : «Quirinus, qui est le dieu des citoyens, [...] présente en son sein une harmonieuse synthèse des trois fonctions » (Briquel, 2003). Sur ce point, voir principalement Briquel, 1995 et surtout Briquel, 1996.

33. Dumézil, 1966, p. 260.

34. Ernout et Meillet, 1932, sv. uir; Delamarre, 2003, sv. uiros.

35 . Le passage de *Querinos à *Queirinus reste toutefois problématique. On peut imaginer une influence du second $i$ sur le $e$ de ${ }^{*} Q u e r i-$, sans que cela soit démontrable. 
tongue archaïque $e i$ en $i^{36}$. En tenant compte de la transformation, dans les langues latines, d'un $p$ - initial en $k w$-, par attraction du deuxième $-k^{w}$, comme dans le cas de quercus (« chêne »), on peut proposer un ancien ${ }^{*} \operatorname{Per}\left(k^{w}\right)$ inus, puis *Querquinus, ce qui nous fait revenir à l'hypothèse de $\operatorname{Cook}^{37}$.

Quirinus pourrait alors appartenir à une longue série de théonymes bien étudiés par divers linguistes, issus de *per-/*per-g-; « frapper » : thrace Perkos ${ }^{38}$; peut-être grec Phorcys $<$ Porkos $^{39}$; norois Fjörgyn (mère de Thor, la Terre) ou Fjörgynn (amant de Frigg) ${ }^{40}$; langues baltes Perkunas (lith.), Perkons (let.), Percunis (prus.) (dieu du Tonnerre); sanskrit Parjanya (épithète probable d'Indra, dieu du

36. Niedermann, 1997 , p. 58 . Cette réduction de la diphtongue produit normalement un i long. Or dans Quirinus, le premier i est court. Cependant, ce type d'argument n'est pas forcément valide dans le cas des théonymes : on a un phénomène exactement similaire avec le slave Perun, dont le nom est accentué sur la deuxième syllabe, alors qu'en russe et dans les autres langues slaves de l'est, perun : «tonnerre », simple nom propre, est accentué sur la première, ce qui n'a pas de justification étymologique! Sur ces variantes: Conte, 1997, p. 86 .

37. Toujours dans le domaine italique, un ethnonyme Quarqueni est bien attesté chez les Vénètes : Ivanov, 1958, p. 102. Faut-il y voir " Ceux du Chêne » ou les «Frappeurs »? Du fait de sa ressemblance avec ce mot, on ajoute régulièrement à la liste l'ethnonyme celtibère des Querquerni (province de Braga : Pline, Histoire naturelle, 3, 28). À Rome même, les Querquetulanae virae désigne chez Festus des Hamadryades : De verborum significatione, 261. Or chez d'autres peuples indo-européens, la racine perg-entre aussi en composition pour former le nom de nymphes ou des déesses-mères : les Nymphes Percernes chez les Voconces, en Gaule (NYMPHIS AUG (ustis) PERCERNIBUS, CIL XII 1329, à Vaisonla-Romaine); les Matrones ou Nymphes Alaferhuiae, dans les Germanies (CIL XIII 12012; 7862; AE 1984 674-692; AE 1926 66; à Bonn, Altdorf, Dormagen et Patteren. Les variantes graphiques de ce théonyme montrent une phonétique en pleine évolution. Ainsi à Patteren, le nom s'écrit Alapherhui [abus], souvenir d'un ancien *Ala-Perhuiabus, alors qu'à Bonn on a un Alaferchuiabus qui trahit un ancien *Ala-ferkuiabus. Il est aisé alors de reconstituer *Ala-Perkuiabus); ou les Matrones Fernovineae (Matronis Fernovineis, CIL XIII 7980, Meckenheim, Allemagne; Fern [ovi] neh [is], AE 1956 246, Cologne, Allemagne). Ces parallèles montrent qu'il a dû exister une riche mythologie autour de la racine perg-à Rome et en Italie. On notera enfin que si les Sabins connaissent curis pour «lance », le mot quercus en latin a pu avoir le même sens : Valérius Flaccus, Argonautiques, VI, 253. On peut alors penser que ces deux mots ont un lien étymologique avec toujours la même racine, la lance étant alors la «frappeuse », sens attendu pour une arme de jet.

38. IG Bulg. I, 283 et 283bis, à Galata (Bulgarie) et Kajalâka (Bulgarie).

39. Alcman, fragment 1, 19. Voir Haggerty Krappe, 1932. et Calame, 1977, t. II, p. 61-62 pour l'étude de Porkos chez Alcman.

40. Haggerty Krappe, 1932, p. 87. 
tonnerre ${ }^{41}$ ); mordvin Purgine (tonnerre), Purgine-pas (dieu du tonnerre) et bien entendu tous les dérivés du Perunъ slave. N'ont été retenus ici que les théonymes majeurs. L'ensemble des dérivés a été bien analysé par Julius Pokorny et Vjačeslav V. Ivanov. Ce dernier auteur permet d'ailleurs de fournir un strict parallèle grec à notre cas avec Keraunos, épithète de Zeus « tonnant ». Ce rapprochement n'est pas reconnu par les spécialistes de l'étymologie grecque ${ }^{42}$. Cependant, Roman Jakobson pense à un primitif *Peraunós et Gregory Nagy admet un étymon *per $(k w)$ aunos, avec une métathèse due à un tabou, donnant $* k w e r(p)$ aunos $^{43}$.

On pourrait objecter que *Querquinus n'est pas *Querinus et que la disparition du second $-k^{w}$ - ne s'explique pas, d'autant plus qu'il est conservé dans quercus. Cependant, dans quasiment tous les parallèles indo-européens signalés ci-dessus, le même phénomène est avéré. Ainsi, les Lithuaniens connaissent à la fois Perkunas et Perunas $^{44}$. Les Slaves ont Perun, mais le lieu de son sanctuaire est Peregynia, et quand les Mordvins leur ont emprunté ce dieu, ils l'ont fait sous le nom de Purgine-pas, preuve qu'il a dû exister un ancien *Pergun. En Inde, alors qu'on attendrait *Parganya (cf. penjabi pargai : " chêne sacré »), on a Parjanya ${ }^{45}$, tout comme chez les Kafirs, Perun, et non *Pergun, désigne le dieu de la guerre ${ }^{46}$. Enfin, nous avons ci-dessus le cas grec de Keraunos. Il semble donc que l'amuissement du $-k^{w}$ - ne s'explique pas par une règle phonétique, mais bien par un ancien tabou d'ordre religieux.

Ce tabou indo-européen, ici peut être lié à l'hellénisation du panthéon latin, et donc à l'identification de Jupiter à Zeus, confirmant au premier le rôle de dieu tonnant, a pu faciliter l'attraction phonétique et sémantique de curia sur *Querinus, du fait d'une perte de sens de ce dernier nom. Autrement dit, *Querquinus serait devenu *Querinus par tabou religieux, puis, au fil de son évolution linguistique supposée $\left({ }^{*}\right.$ Querinus $>$ Queirinus $)$, le mot aurait perdu son

41. Indra et Parjanya sont, a priori, dans les Védas, des dieux indépendants, mais leurs hymnes, leurs actions, leur mythologie sont en tous points similaires et on peut voir en Parjanya un aspect d'Indra.

42. En dernier lieu : Chantraine, 2000, sv. Kepauvoc. Il vient cependant d'être admis par West, 2007, p. 243-244.

43. Jakobson, $1985 a$ et $b$; Nagy, 1974, p. 128.

44. Jakobson, $1985 a$ et $b$.

45. West, 2007, p. 245.

46. Jakobson, 1985a, p. 20; Gamkrelidze et Ivanov, 1995, p. 528. 
sens immédiatement intelligible, avant d'être raccroché, par étymologie populaire, à curia et à ses dérivés. L'hypothèse de cette ultime étape, qui a pu avoir lieu au tournant du $\mathrm{II}^{\mathrm{e}}$ siècle avant J.-C., trouve un appui dans le cas samnite, où l'on voit Queirinus encore associé à Hercule, fils de Jupiter, et au foudre.

\section{Un ancien dieu du tonnerre?}

Dans la version anglaise de leur grand essai sur la proto-culture indo-européenne, Thomas Gamkrelidze et Vjačeslav Ivanov avaient postulé l'existence d'un binôme céleste dont l'un des membres est le ciel lui-même (Dyaus, Odin, Dievas, etc.) et l'autre un dieu tonnant et combattant, subordonné (Indra, Thor, Perkunas, etc.). Parfois, ce binôme pose de stricts équivalents linguistiques : Dyaus/Parjanya; Dievas/Perkunas par exemple. Même lorsque les noms sont oubliés, ce binôme existe : Odin et Thor; Dagda et $\mathrm{Lug}^{47}$. Mais, comme le notent Thomas Gamkrelidze et Vjačeslav Ivanov, en Grèce et en Italie, le dieu céleste (Zeus et Jupiter) et un autre dieu guerrier (Arès et Mars) ont récupéré tout ou partie des attributs du dieu tonnant ${ }^{48}$. Il est donc tout à fait possible qu'un tabou, en Grèce et à Rome, ait agi sur le nom de ce dernier dieu. La différence étant qu'en

47. En Irlande, le cas est moins net que dans les pays scandinaves où Odin a récupéré sans doute bon nombre d'attributs de Tyr $(*$ Tywaz). Cependant il semble bien que le Dagda (le « Bon Dieu ») n'a que peu de caractères tonnants alors que Lug en conserve dans certaines légendes : Sergent, 2004, p. 99. Ce fait ne doit pas surprendre et il est possible que cela ait été aussi le cas en Gaule avant la conquête romaine. Ainsi, quand Lucain parle d'Esus, Taranis et Toutatis, il se pourrait qu'on ait affaire à une triade similaire à Jupiter, Quirinus et Mars. Esus signifie « Dieu » (de la même manière que Jupiter est « Dieu père »), Taranis est le « Tonnerre » et Toutatis a été systématiquement assimilé à Mars à l'époque gallo-romaine. Pour une première tentative de comparaison de ce type : Dumézil, 1947, p. 113. Mais ce type de triade n'a, en définitive et nous le verrons plus bas, rien de trifonctionnel. Pour une réfutation de l'hypothèse de Georges Dumézil, mais avec d'autres solutions : Steckx, 2005, p. 422, n. 89.

48. Gamkrelidze et Ivanov, 1995, p. 697-699. Ces deux auteurs acceptent les fonctions agraires prêtées à Mars. On peut ajouter que Mars a aussi peut-être récupéré le chêne oraculaire: Denys d'Halicarnasse, Antiquités romaines, I, XI. Cf. Haggerty Krappe, 1932, p. 79. L'hypothèse de Gamkrelidze et Ivanov est reprise sans changements par West, 2007. Pisani, 1950, p. 10, élude la question en faisant de Perk ${ }^{w} u n o s$, dieu tonnant, une simple hypostase de Dyaus, dieu céleste. On notera quand dans le monde gréco-romain, ce sont justement Jupiter et Mars (ou Zeus et Arès) qui se voient attribués le chêne, alors que partout ailleurs cet arbre est l'apanage exclusif du dieu du tonnerre. 
Grèce ce nom est devenu épithète de Zeus, Kéraunos (encore qu'il se soit peut-être aussi réfugié sous une autre forme dans Phorkys), alors qu'en Italie et à Rome, il est resté un dieu indépendant mais méconnu, Quirinus.

Quirinus n'a quasiment plus d'iconographie. Sa statue n'est pas connue. Pas contre, il en reste un portrait frappé sur des deniers de diverses époques (denier de C. Memmius, 56 avant J.-C.; denier de Trajan $)^{49}$. Le portrait en question est plutôt surprenant, car au lieu d'un dieu juvénile, qu'on serait en droit d'attendre du fait qu'il serait fils de Mars (par son assimilation à Romulus), lui-même dieu jeune, on voit un personnage mature, barbu, à tête laurée : ce portrait aurait pu être celui de Jupiter ${ }^{50}$.

Cependant, on ne peut tirer de conclusions de ce type d'argument. Plus intéressante est l'iconographie du sanctuaire d'Hercule Quirinus à Sulmona. La mosaïque qui pave la cella du sanctuaire est faite de carrés concentriques entourés d'une frise de dauphins puis de vigne. Légèrement excentrée, à l'opposé des statues par rapport à la mosaïque, se trouve la représentation d'un grand faisceau de foudre, attribut habituel de Jupiter ${ }^{51}$ !

Enfin, si Quirinus n'a plus de mythologie, reste celle de Romulus, dont tous les auteurs antiques disent qu'il est Quirinus incarnés2. Denys d'Halicarnasse le martèle presque : «Le génie qui m'a été attribué à ma naissance me conduit aux dieux, maintenant que j'ai fini ma vie mortelle, et que je suis Quirinus », fait-il dire à Romulus $^{53}$.

\section{Des JuMeauX}

Le père de Romulus et Rémus serait Mars : c'est un fait quasi unanimement reconnu par les auteurs antiques. Si diverses variantes coexistent sur cette paternité, l'iconographie, elle, atteste unique-

49. LIMC, VII, sv. Quirinus.

50. Selon Suétone, Tibère portait une couronne de laurier, "parce que la feuille de cet arbre est, dit-on, à l'abri de la foudre »! Suétone, Tibère, LXIX.

51. http://www.soprintendenza-archeologica.ch.it/ErcoleCurino.htm

52. Liou-Gille, 1980 , p. 141, suivant d'autres auteurs, montre bien que l'assimilation remonte au moins au $\mathrm{III}^{\mathrm{e}}$ siècle. Nous ne possédons pas de témoignages antérieurs faute de sources littéraires.

53. Antiquités romaines, II, 63, 4. 
ment d'une fécondation de Réa Silvia durant son sommeil ${ }^{54}$. Contrairement à ce qu'a pu dire Georges Dumézil, la gemmélité n'est pas caractéristique de la troisième fonction ${ }^{55}$.

Cependant, il est clair qu'il faut distinguer deux types de jumeaux indo-européens. Dans les cas où les deux frères sont immortels (comme les Aśvin), ils sont effectivement typiquement de troisième fonction. Mais lorsque l'un des deux frères est mortel, on peut constater que tous les deux sont guerriers. Romulus et Rémus sont des combattants, et Rémus meurt tué par son propre frère. De même, peu de choses chez les Dioscures permettent de dire qu'ils appartiennent à la troisième fonction. Or des deux frères, Castor est mortel car Zeus n'est père que de Pollux ${ }^{56}$. Toujours en Grèce, Héraklès est un jumeau célèbre : s'il est né de Zeus, son frère Eurysthée est issu d'Amphitryon, et par là donc, est mortel. Chez les Celtes, Lug est aussi un jumeau, mais que ce soit en Irlande ou au Pays de Galles, ses frères meurent (ou disparaissent) dans la mer. On ne peut nier à Lug ou à Héraklès leur appartenance à la fonction guerrière.

\section{L'ABANDON DANS LES EAUX}

Romulus et Rémus sont ensuite placés dans une corbeille et laissés sur le Tibre. Cet abandon aux eaux est un motif très fréquent en mythologie. Mais on peut noter qu'il est régulièrement accolé à des personnages guerriers, voire tonnants. En Inde, on attribue cet abandon à Indra, dieu de la guerre et de l'orage, peut-être surnommé Parjanya, comme nous l'avons vu ${ }^{57}$. En Grèce, c'est Persée, fils de Zeus, qui en fait l'objet, mais on peut noter qu'Héraklès a aussi fait l'objet d'une exposition, d'un abandon. Chez les Celtes irlandais,

54. LIMC, VII, sv. Rhea Silvia. Une variante intéressante fait de Romulus et Rémus les fils d'une servante de la fille du roi des Albains Tarchétius et d'un phallus miraculeux, sorte de dieu masculin du foyer : Plutarque, Romulus, II. Cette version est très proche de celle qu'on connaît sur la naissance de Caeculus à Préneste, qui semble fils de Vulcain (ou tout au moins d'une étincelle issue d'un foyer), ainsi que sur Servius Tullius, sixième roi de Rome. Il est possible que cette version soit (donc) simplement due à la popularité de ce genre de motifs chez les peuples italiques.

55. Dumézil, 1966, p. 252-255. Il est largement suivi par Sergent, 1997, p. 315-316. Mais des jumeaux n'appartenant pas à la troisième fonction ont déjà été repérés par York, 1988, p. 153-154. Mais celui-ci a trop élargi sa problématique au duo Mitra-Varuna.

56. Pindare, Néméennes, $\mathrm{X}, 81$ et suiv.

57. Rg Veda, 4, $18:$ La Naissance d'Indra. 
c'est encore Lug qui est jeté à la mer, avec ses frères. En Espagne, l'un des premiers rois de Tartessos, Gargoris, subit le même sort ${ }^{58}$. Cependant, on a perdu la fin de sa légende, mais comme le nom même du personnage, probablement celtique, est fondé sur gargo- : " féroce, sauvage », on peut penser que lui non plus n'appartient pas à la troisième fonction ${ }^{59}$. Or comme Romulus, c'est un fondateur de lois. En Russie, lors de la christianisation, ce n'est sans doute pas un hasard si on décide de se débarrasser des statues de Perunъ en les jetant dans l'eau d'un fleuve, que ce soit à Kiev ou à Novgorod. On pourrait encore multiplier les exemples.

\section{Apothéose tonnante}

Mais en dehors d'une vie de fondateur de cité somme toute classique, ce qui marque le plus dans le personnage de Romulus est son apothéose. Pas un auteur classique ne nie l'identification de Romulus à Quirinus ${ }^{60}$. Et le récit de son apothéose est pour le moins étonnant ${ }^{61}$. Celui qu'en fait Ovide est un des plus circonstancié : Mars demande à Jupiter, son père, de pouvoir récupérer son fils survivant. Jupiter accepte. À un moment où Romulus est en train de rendre la justice dans le marais de la Chèvre, «Le soleil disparait, des nuages s'avancent, qui dissimulent le ciel, et une averse s'abat lourdement, avec des torrents d'eau. Ici gronde le tonnerre; là, des éclairs répétés déchirent l'éther; sauve-qui-peut général; le roi gagnait le ciel sur les chevaux de son père ${ }^{62} »$. Ovide détaille encore plus dans les Métamorphoses : « le dieu l'enlève, et ce que son corps eut de mortel s'évanouit dans les airs, comme la balle de plomb lancée par la fronde, s'embrase et se perd dans la nue ${ }^{63} »$. La $1-14$

58. Justin, Abrégé des Histoires Philippiques de Trogue Pompée, XLIV, IV,

59. Delamarre, 2003, sv. gargo-.

60. Seul Cicéron admet que tous n'y croient pas : Schilling, 1999, p. 1739.

61. Ce type d'apothéose est très fréquent dans le monde hellénistique, au point même de se retrouver inséré dans la Bible, au sujet de saint Élie. Mais quand bien même il s'agirait d'un emprunt par les Romains, cet emprunt n'a pu se faire inutilement, par simple « goût littéraire » : il devait avoir un sens religieux. On notera par ailleurs que parmi les versions alternatives, Romulus serait mort de la main de sénateurs, mais on n'aurait jamais retrouvé son corps.

62. Ovide, Fastes, 2, 475-532. Florus, I, 1, ajoute qu'il y eut une éclipse du soleil.

63. Métamorphoses, XIV, 816 et suiv. 
balle de fronde est une belle évocation de la foudre : en Irlande, Lug tue Balor d'une balle de fronde incandescente et, en Scandinavie, Thor abat un géant de la même manière ${ }^{64}$. Effectivement, l'enlèvement de Romulus a lieu durant un orage ${ }^{65}$, un orage qu' on ne dit pas être provoqué par Jupiter, lequel est passif. Mais l'élément le plus intéressant est la présence d'un char.

\section{Quirinus et Mars}

Certes, il n'y a pas lieu de faire de Quirinus et de Mars les équivalents divins de Romulus et Rémus, et donc par conséquent des jumeaux ${ }^{66}$. Quirinus est représenté comme un homme mûr, barbu, majestueux, ce qui n'est pas le cas de Mars, juvénile. Sur l'inscription de Rome, Quirinus vient avant Mars. En ajoutant que Mars sert de cocher à Quirinus lors de l'apothéose, il devient légitime de penser que, dans un état perdu du mythe, Mars était subordonné à Quirinus.

On peut alors revenir sur un ancien problème linguistique : celui de l'étymologie de Mars. A. Ernout et A. Meillet lui ont dénié toute étymologie indo-européenne ${ }^{67}$. Pourtant, les diverses formes archaïques ou dialectales qui lui sont attestées ${ }^{68}$ ont conduit certains spécialistes à tenter un rapprochement avec les Maruts védiques. Ce rapprochement, fut admis dans un premier temps par Georges Dumézil, avant d'être mis en doute ${ }^{69}$ puis fermement rejeté ${ }^{70}$. Il est pourtant toujours reconnu comme valide par divers linguistes ${ }^{71}$.

64. Van Berg, 2006, p. 296-297. Cela n'est d'ailleurs pas la première fois que Romulus a affaire à la foudre: selon le pseudo-Aurélius Victor, Les Origines $d u$ peuple romain, XXIII, 2-3, lorsque Romulus et Rémus choisirent d'en appeler aux auspices pour savoir lequel sera le fondateur de la ville, il apparait à Rémus six vautours, alors que Romulus en voit douze, accompagnés d'un éclair et du bruit du tonnerre.

65. Ce qui n'est pas sans rappeler l'enlèvement au ciel du prophète Élie, dans l'Ancien Testament; Élie qui fut un grand destructeur du culte du dieu de l'orage de Canaan, Baal.

66. Contrairement à York, 1988.

67. Ernout et Meillet, 1932, sv. Mars.

68. Mauort-, Maurs, Marmar, osque Mamers.

69. Dumézil, 1966, p. 214-215.

70. Dumézil, 2003, p. 455 : «à plus forte raison, je n'ai aucun plaisir à voir aujourd'hui relever et défendre, au prix d'acrobaties phonétiques, les étymologies dont j'étais parti dans les dérèglements (ou l'absence de règle) des débuts et que j'ai, par la suite, explicitement rejetées »!

71. Haudry, 1981, p. 80; Prat, 1992, p. 120. Le mot Marut a peut-être subi l'influence de māra : "mort », sans y être étymologiquement lié. Chez les Slaves, 
Le rapprochement semble d'autant plus intéressant que si, à Rome, les Salii dansent, en armes, pour célébrer Mars (ou Quirinus, qui avait ses propres Salii), les Maruts sont aussi des danseurs ${ }^{72}$. Il est d'autant plus significatif qu'en Inde, les Maruts sont, bien que guerriers et personnifications des vents, les chefs de la classe ouvrière des agriculteurs-éleveurs, selon les Brâhmanas ${ }^{73}$. Or les Maruts sont surtout les compagnons d'Indra. De fait, sauf encore une fois en Grèce et en Italie, les dieux de l'orage indo-européens semblent être entourés d'esprits des airs. Dans le folklore irlandais, c'est en compagnie des «étincelles de Balor» que Lug vole dans les airs pendant l'orage ${ }^{74}$. Chez les Baltes, Perkunas/Perkons est doté de plusieurs fils, du même nom, qui ont exactement les mêmes fonctions que lui ${ }^{75}$. En Roumanie, où, comme dans tous les pays orthodoxes, le prophète Élie a remplacé le dieu de l'orage, il lutte contre le Diable précédé par des anges, qui fendent les nuages ${ }^{76}$. Mais on dit aussi qu'au lieu d'anges, ce serait des Zmei (dragons), lesquels peuvent produire la pluie, la sécheresse ou le vent : quand deux Zmei se rencontrent, ils se battent et produisent des éclairs ${ }^{77}$. Dans le Caucase, Élie apparaît d'ailleurs sous une forme plurielle, les Wacillatæ, qui sont des démons aériens, ennemis de Batraz, sans doute un ancien dieu tonnant des Ossètes ${ }^{78}$. En Scandinavie, Odin, dieu complexe, a récupéré sur sa personne des éléments de diverses divinités, c'est donc lui, et non Thor, qui mène la chasse sauvage. Mais celle-ci reste malgré tout liée aux tempêtes ${ }^{79}$. En fin de compte, plutôt qu'une optique trifonctionnelle, avec le dossier Quirinus/Mars, on peut opter pour une optique du type Indra/Vayu, c'est-à-dire la force contrôlée et la force débridée. C'est sans doute pour cela que Quirinus est compris tardivement comme un Mars pacifique.

les Maruts encadrant Indra sont remplacés par les Zmeï encadrant saint Élie « le tonnant». Or les Zmeï sont, étymologiquement et bien qu'étant des esprits atmosphériques, « Ceux de la Terre (Zemlia)».

72. Dumézil, 1966, p. 213. Le nom même des Salii a été rapproché de celui des Helloi, les premiers prêtres desservant le sanctuaire de Zeus à Dodone.

73. Daniélou, 1994, p. 167.

74. Sergent, 2004, p. 99.

75. Jouet, 1989, p. 112, par exemple.

76. Talo ş, 2002, p. 78.

77. Ibid., p. 74-75. On notera que les Zmei sont censés être issus de Balaur : la coïncidence de nom avec le compagnon aérien de Lug est troublante.

78. Dumézil, 1978, p. 74 et suiv.

79. Lecouteux, 1999, p. 183-187. 


\section{Mars et Quirinus agraires?}

Mais tous les personnages cités ci-dessus à titre de comparaison sont aussi liés aux récoltes et à leur protection. Dès le Moyen Âge, Thor est le protecteur des récoltes, ce qui se retrouve aussi en Suède septentrionale, où il est « le bon paysan; le bonhomme du blé; des champs $^{80} »$. Chez les Baltes, on prie Perkunas/Perkons pour qu'il fasse tomber la pluie, de façon à ce que les jeunes pousses ne dessèchent pas. Les Mordvins font de même pour Purgine-pas et son avatar chrétien Il'ja (Élie), tous deux empruntés aux Slaves. On remarquera au passage que chez les Hittites, Tešub, dieu de l'orage, a pour fils Telepinu, dieu de la végétation (lequel est aussi capable de lancer des éclairs). Lorsque celui-ci est en colère, il tonne, s'en va et le pays se dessèche ${ }^{81}$. En Inde, donc, les Maruts appartiennent à la classe des agriculteurs-éleveurs.

Cela revient à dire que la question d'un Mars ou d'un Quirinus « agraire » est un faux problème, si on accepte la théorie de Quirinus comme ancien dieu tonnant ${ }^{82}$. Dans le monde indo-européen et même au-delà, tous les dieux de l'orage sont liés à la fécondité, aux récoltes : ils en sont les protecteurs, protection effectuée par les armes. Le dieu de l'orage est donc guerrier, mais par ses aptitudes, il protège la troisième fonction indo-européenne, fonction reproductrice, et non fonction agricole $\mathrm{e}^{83 *}$.

patrice.lajoye@unicaen.fr

80. Ibid., p. 186.

81. Mazoyer, 2003. On remarquera aussi que tous ces dieux (ou successeurs de dieux, comme Élie) traversent le ciel en char, char que l'on retrouve, donc, dans le récit de l'apothéose de Romulus chez Ovide.

82. En analysant le personnage de Romulus, É. Tiffou se tire d'affaire en faisant de celui-ci un roi trifonctionnel : Tiffou, 1976. Cela pourrait être intéressant car cette théorie validerait la comparaison avec l'irlandais Lug, lui-même hors fonction. Georges Dumézil, à la fin de sa carrière, commençait à admettre un « glissement vers un statut de guerrier» : Dumézil, 1996, p. 165.

83. Sergent, 2004, p. 283-284. -

* J'adresse mes plus vifs remerciements à la Revue de l'histoire des religions : le premier rapporteur qu'elle a désigné m'a permis d'améliorer grandement mon argumentaire et la relecture de détail de M. Jean-Michel Roessli (Université de Sudbury, Canada) m'a été très bénéfique. 


\section{BIBLIOGRAPHIE}

Vsevolod Basanoff, Les Dieux des Romains, 1942, Paris, PUF.

Claude Calame, Les Chours de jeunes filles en Grèce archaïque, II, Alcman, 1977, Rome, Edizioni dellAteneo \& Bizzarri.

Dominique Briquel, «Le problème d'Ancus Marcius », Mélanges de l'Ecole française de Rome (Antiquité), 107, 1995, p. 183-195.

Dominique BrIQuel, « Remarques sur Quirinus », Revue belge de philologie et d'histoire, 74, 1996, p. 99-120.

Dominique Briquel, « Tullus Hostilius et le thème indo-européen des trois péchés du guerrier $»-1^{\mathrm{re}}$ partie, Folia Electronica Classica, 5, 2003, http://bcs.fltr.ucl.ac.be/FE/05/tullus1.html\#499138.

Francis CONTE, L'héritage païen de la Russie. Le paysan et son univers symbolique, 1997, Paris, Albin Michel.

Arthur Bernard Cook, "Zeus, Jupiter, and the oak - conclusion », The Classic Review, XVIII, 1904, p. 360-374.

Alain DaniéLou, Mythes et dieux de l'Inde, 1994, Paris, Flammarion, «Champs ».

Delamarre Xavier, Dictionnaire de la langue gauloise, 2003, Paris, Errance.

Georges DumÉzIL, Jupiter, Mars, Quirinus, 1941, Paris, Gallimard.

Georges DumÉzIL, Naissance de Rome, 1944, Paris, Gallimard.

Georges DumÉzIL, Tarpéia, 1947, Paris, Gallimard.

Georges DumézIL, Explications de textes, 1948, Paris, Gallimard.

DumÉzIL Georges, L’Héritage indo-européen à Rome, 1949, Paris, Gallimard.

Georges DumÉzIL, La Religion romaine archaïque, 1966, Paris, Payot.

Georges DumÉzIL, Romans de Scythie et d'alentour, 1978, Paris, Payot.

Georges DumézIL, Mythes et épopée, 1995, Paris, Gallimard, « Quarto ».

Georges DumÉzIL, Heur et malheur du guerrier, 1996, Paris, Flammarion, « Champs ». 
Georges DumÉzIL, Esquisses de mythologie, 2003, Paris, Gallimard, «Quarto ».

Alfred Ernout et Antoine MeIllet, Dictionnaire étymologique de la langue latine. Histoire des mots, 1932, Paris, Klincksieck.

James Georges Frazer, Le Roi magicien dans la société primitive, vol. II, 1935, Paris, Geuthner.

Thomas V. Gamkrelidze et Vjačeslav V. Ivanov, Indo-European and the Indo-Europeans. A Reconstruction and Historical Analysis of a ProtoLanguage and a Proto-Culture, 1995, Berlin/New York, Mouton de Gruyter.

Alexandre Haggerty Krappe, «Les Péléiades », Revue archéologique, 1932, V, 36, p. 77-93.

Jean Haudry, Les Indo-Européens, 1981, Paris, P.U.F., « Que sais-je? ».

Jean HAUdRY, « La préhistoire de Janus », Revue des études latines, t. 83, 2006, p. 36-54.

Lauri Honko, Senni Timonen, Michael Branch et Keith Bosley, The Great Bear. A Thematic Anthology of Oral Poetry in the Finno-Ugrian Languages, 1993, Pieksämäki, Finnish Literature Society Editions.

Vjačeslav V. IvANOv, « K etimologii baltijskogo i slavjanskogo nazvanij boga groma » («Sur l'étymologie du nom balte et slave du dieu du tonnerre »), Voprosy Slavjanskogo Jazykoznanija, 3, 1958, p. 101-111.

Vjačeslav V. Ivanov et Vladimir N. ToPOROV, « Le mythe indo-européen du dieu de l'orage poursuivant le serpent : reconstruction du schéma », in Jean Pouillon (dir.), Échanges et communications. Mélanges offerts à Claude Lévi-Strauss, 1970, Paris/La Haye, Mouton, p. 1180-1206.

Roman JAKOBSON, « Slavic gods and demons », in Selected Writings, VII, 1985a, Mouton, La Haye/Paris, p. 3-32.

Roman JAKOBSON, "The Slavic God Velesb and his Indo-European Cognates », in Selected Writings, VII, 1985b, Mouton, La Haye/Paris, p. 33-48.

Philippe Jouet, Religion et mythologie des Baltes, 1989, Milan/Paris, Archè/Les Belles Lettres.

Paul Kretschmer, «Lat. quirites und quiritare », Glotta, 10, 1920, p. 147-157. 
Claude Lecouteux, Chasses fantastiques et cohortes de la nuit au Moyen Age, 1999, Paris, Imago.

Bernadette Liou-Gille, Cultes "hérö̈ques 》 romains. Les fondateurs, 1980, Paris, Les Belles Lettres.

Michel Mazoyer, Télipinu, le dieu au marécage. Essai sur les mythes fondateurs du royaume hittite, 2003, Paris, L'Harmattan.

Gregory Nagy, « Perkúnas and Perunъ », in Manfred Mayrhofer, Wolfgang MeID et alii, Antiquitates indogermanicae, 1974, Innsbruck, « Innsbrücker Beiträge zur Sprachwissenschaft», XII, p. 113-131.

Max Niedermann, Phonétique historique du latin, 1997, Paris, Klincksieck.

Ivar Paulson, Åke Hultkrantz et Karl Jettmar, Les Religions arctiques et finnoises. Sibériens, Finnois, Lapons, Esquimaux, 1965, Paris, Payot.

Vittore PisAnI, Le religioni dei Celti e dei Balto-Slavi nell'Europa Precristiana, 1950, Milan, Istituto Editoriale Galileo.

Julius PoKORNY, Indogermanisches Etymologisches Wörterbuch, 1958, Berne et Munich, Francke Verlag.

Danielle PorTe, «Romulus-Quirinus, prince et dieu, dieu des princes. Étude sur le personnage de Quirinus et sur son évolution, des origines à Auguste », Aufstieg und Niedergang der römischen Welt, 1981, II, 17.1 p. 300-342.

Louis Prat, Mours, civilisation et idéologie des Indo-Européens, 1992, Paris, Klincksieck.

Aldo L. Prosdocimi, « Curia, Quirites e il 'sistema di Quirino' (Populus Quiritium Quirites II) », Ostraka, 1996, n 2, p. 243-319.

Gerhard RADKE, « Quirinus. Eine kritische Überprüfung der Überlieferung und ein Versuch », Aufstieg und Niedergang der römischer Welt, 1981, II, 17.1 , p. 276-299.

Robert Schilling, «Quirinus », in Yves Bonnefoy, (dir.), Dictionnaire des mythologies, 1999, Paris, Flammarion, t. 2, p. 1738-1740.

Bernard Sergent, Les Indo-Européens. Histoire, langues, mythes, 1995, Paris, Payot.

Bernard Sergent, Genèse de l'Inde, 1999, Paris, Payot. 
Bernard SERGENT, «La troisième fonction : la fertilité ou l'abondance », General Linguistic, 41, nº 1-4, 2004 (2001), p. 270-289.

Bernard Sergent, Le Livre des dieux. Celtes et Grecs II, 2004, Paris, Payot.

Claude Steckx, Taranis, Sucellos et quelques autres. Le dieu souverain des Celtes, de la Gaule à l'Irlande, 2005, Bruxelles, Mémoires de la Société Belge d'Etudes Celtiques, 24.

Ion Taloș, Petit dictionnaire de mythologie populaire roumaine, 2002, Grenoble, Ellug.

Étienne Tiffou, «Notes sur le personnage de Romulus », Mélanges offerts à Jacques Heurgon, 1976, Rome, École française de Rome, t. II, p. 991-999.

Paul-Louis van BERG, «L'Eil et le Bras plutôt que le Borgne et le Manchot : une solution alternative à la proposition de Georges Dumézil », in Marco V. Garcia Quintela, Francisco J. González García et Felipe Criado BoAdo (éd.), Anthropology of the Indo-European World and Material Culture. Proceedings of the $5^{\text {th }}$ International Colloquium of Anthropology of the Indo-European World and Comparative Mythology, 2006, Budapest, Archaeolingua, p. 285-304.

Martin L. West, Indo-European Poetry and Myth, 2007, Oxford, Oxford University Press.

Michael York, « Romulus and Remus, Mars and Quirinus », Journal of Indo-European Studies, 1988, 16, p. 153-172. 